\title{
The GoPA! Second Set of Country Cards Informing Decision Making for a Silent Pandemic
}

\author{
Andrea Ramírez Varela and Michael Pratt
}

\begin{abstract}
In 2012, the Global Observatory for Physical Activity (GoPA!) was established to provide information that would enable countries to initiate or improve research capacity, surveillance systems, program development, and policymaking to increase physical activity levels. Findings from the first GoPA! Country Cards showed an unequal distribution of physical activity surveillance, research productivity, and policy development and implementation around the world. Regular global monitoring of these factors, especially in countries with the largest data gaps, was recommended to combat the global pandemic of physical inactivity. After 6 years and using standardized methods, GoPA! is launching the second set of Country Cards based on data up to 2019 from 217 countries. Overall results showed that periodic national surveillance of physical activity was less common in lowincome countries, compared with middle- and high-income countries. Large inequities were seen with more than a 50-fold difference in publications between high- and low-income countries and $32 \%$ of the countries worldwide had no physical activity policy. GoPA! has a critical role in facilitating evidence-based physical activity promotion building on international guidelines and the World Health Organization Global Action Plan. GoPA! will continue to monitor progress as we battle the global pandemic of physical inactivity.
\end{abstract}

Keywords: research, epidemiology, public health, surveillance

The landmark article about physical activity and health was published in $1953 .{ }^{1}$ Since its publication, evidence supporting the beneficial role of physical activity for health has grown exponentially, and over the last 2 decades, public health efforts to increase population levels of physical activity have increased as well. ${ }^{2}$ However, one of every 3 adults worldwide remains physically inactive. $^{3}$ In 2012, the Global Observatory for Physical Activity (GoPA!) ${ }^{4}$ was established to provide information that would enable countries to initiate or improve research capacity, surveillance systems, program development, and policymaking to increase physical activity levels. ${ }^{2,5,6}$ Findings from the first GoPA! Country Cards showed an unequal distribution of physical activity surveillance, research productivity, and policy development and implementation around the world. ${ }^{2}$ Regular global monitoring of these factors, especially in countries with the largest data gaps, was recommended to combat the global pandemic of physical inactivity.

After 6 years and using standardized methods, GoPA! is launching the second set of Country Cards based on data up to 2019 from 217 countries. Overall results showed that $187(86.2 \%)$ of countries had at least 1 national survey that assessed physical activity prevalence, $147(67.7 \%)$ had some degree of ongoing national physical activity surveillance (at least 2 surveys), 30 $(13.8 \%)$ had no surveillance, but only 27 (12.4\%) countries had a plan for the next national survey. Periodic national surveillance of physical activity was less common in low-income countries compared with middle- and high-income countries. Since the 1950s, $175(80.6 \%)$ countries had at least 1 peer-reviewed research publication. However, large inequities were seen with more than a 50-fold difference in publications between high- and low-income

Ramirez Varela is with the School of Medicine, Universidad de los Andes, Bogotá, Colombia. Pratt is with the School of Medicine, Herbert Wertheim School of Public Health and Longevity Science, University of California, San Diego, San Diego, CA, USA. Ramirez (an-rami2@uniandes.edu.co) is corresponding author. countries. Sixty-six $(30.4 \%)$ countries had a standalone physical activity policy, $81(37.3 \%)$ had a noncommunicable disease policy that included physical activity, and 70 (32.3\%) had no physical activity policy.

When comparing the GoPA! 2015 and 2020 indicators by country income group, research productivity (number of articles) was equal or better for $64(78.0 \%)$ high-income countries $(n=82)$, $49(87.5 \%)$ upper middle-income countries $(n=56), 37(74.0 \%)$ lower middle-income countries $(n=50)$, and $25(86.2 \%)$ lowincome countries $(n=29)$. An equal or increased surveillance capacity (reporting same data as in 2015 or new data for the first, most recent, and next surveys) was found for 64 (78.0\%) highincome countries, $44(78.6 \%)$ upper middle-income countries, 31 $(62.0 \%)$ lower middle-income countries, and $25(86.2 \%)$ lowincome countries. Equal or increased evidence of national policy (reporting the same data as in 2015 or new data for a noncommunicable disease plan including physical activity or a standalone plan) was found for 46 (56.1\%) high-income countries, $35(62.5 \%)$ upper middle-income countries, $22(44.0 \%)$ lower middle-income countries, and $25(86.2 \%)$ low-income countries.

The last decade was a period of great progress in global physical activity surveillance, policy, and research. However, continuously updated information is and will continue to be needed to achieve the World Health Organization global objective of a $15 \%$ relative reduction in the prevalence of physical inactivity among adults by $2030 .^{7,8}$ GoPA! has a critical role to play in facilitating evidence-based physical activity promotion building on international guidelines ${ }^{9}$ and the World Health Organization Global Action Plan. ${ }^{7}$ GoPA! will continue to monitor progress as we battle the global pandemic of physical inactivity.

\section{Acknowledgments}

All the country cards are available in English and in many cases the cards have been translated into national languages. Without the input, review, 
and support of the local representatives/Country Contacts in each of the included countries, the country cards could not have been completed. We also acknowledge important support from the GoPA! Steering Committee: Anita Nguyen from the University of California, San Diego, USA; Cíntia Borges, Paulo Ferreira, and Marcelo Cozzensa of the Federal University of Pelotas, Brazil; Catalina del Portillo from the Universidad de los Andes, Colombia; and the Board of the International Society for Physical Activity and Health.

\section{References}

1. Varela AR, Pratt M, Harris J, et al. Mapping the historical development of physical activity and health research: a structured literature review and citation network analysis. Prev Med. 2018;111:466-472. PubMed ID: 29709233 doi:10.1016/j.ypmed.2017.10.020

2. Ramirez Varela A, Pratt M, Powell K, et al. Worldwide surveillance, policy and research on physical activity and health: the global observatory for physical activity-GoPA! J Phys Act Health. 2017:1-28.

3. Guthold R, Stevens GA, Riley LM, Bull FC. Worldwide trends in insufficient physical activity from 2001 to 2016: a pooled analysis of 358 population-based surveys with 1.9 million participants. Lancet
Global Health. 2018;6(10):e1077-e86. PubMed ID: 30193830 doi:10.1016/S2214-109X(18)30357-7

4. GoPA! Global Observatory for Physical Activity 2016 . http://www. globalphysicalactivityobservatory.com/goals/.

5. Hallal PC, Martins RC, Ramirez A. The lancet physical activity observatory: promoting physical activity worldwide. Lancet. 2014; 384(9942):471-472. PubMed ID: 25110267 doi:10.1016/S01406736(14)61321-0

6. Pratt M, Ramirez A, Martins R, et al. 127 steps toward a more active world. J Phys Act Health. 2015;12(9):1193-1194. PubMed ID: 26684668 doi:10.1123/jpah.2015-0569

7. World Health Organization. Global Action Plan on Physical Activity 2018-2030: More Active People for a Healthier World: At-a-Glance. World Health Organization; 2018.

8. Pratt M, Varela AR, Salvo D, Kohl Iii HW, Ding D. Attacking the Pandemic of Physical Inactivity: What Is Holding Us Back?: BMJ Publishing Group Ltd and British Association of Sport and Exercise Medicine; 2020.

9. Bull FC, Al-Ansari SS, Biddle S, et al. World Health Organization 2020 guidelines on physical activity and sedentary behaviour. $\mathrm{Br} J$ Sports Med. 2020;54(24):1451-1462. PubMed ID: 33239350 doi:10. 1136/bjsports-2020-102955 